\title{
Erratum to: Regioselective Glucuronidation of Andrographolide and Its Major Derivatives: Metabolite Identification, Isozyme Contribution, and Species Differences
}

\author{
Xiangge Tian, ${ }^{1}$ Sicheng Liang, ${ }^{2}$ Chao Wang, ${ }^{1}$ Baojian Wu, ${ }^{3}$ Guangbo Ge, ${ }^{2}$ Sa Deng, ${ }^{1}$ Kexin Liu, ${ }^{1}$ \\ Ling Yang, ${ }^{2}$ and Xiaochi $\mathrm{Ma}^{1,4}$
}

\section{Erratum to: AAPS J 10.1208/s12248-014-9658-8}

The authorship and affiliation for this article should read as follows:

Xiangge Tian, ${ }^{1}$ Sicheng Liang, ${ }^{2}$ Chao Wang, ${ }^{1}$ Baojian $\mathrm{Wu}^{3}$ Guangbo Ge, ${ }^{2}$ Sa Deng, ${ }^{1}$ Kexin Liu, ${ }^{1}$ Ling Yang, ${ }^{2}$ and Xiaochi $\mathrm{Ma}^{1,4}$

${ }^{1}$ College of Pharmacy, Academy of Integrative Medicine, Dalian Medical University, Dalian, 116044, China.

${ }^{2}$ Laboratory of Pharmaceutical Resource Discovery, Dalian Institute of Chemical Physics, Chinese Academy of Sciences, Dalian, China.

${ }^{3}$ College of Pharmacy, Jinan University, Guangzhou, 510632, China.

${ }^{4}$ To whom correspondence should be addressed. (e-mail: maxc1978@163.com)

Xiangge Tian and Sicheng Liang contributed equally to this work.

Xiangge Tian and Sicheng Liang contributed equally to this work. The online version of the original article can be found at http:// dx.doi.org/10.1208/s12248-014-9658-8.

\footnotetext{
${ }^{1}$ College of Pharmacy, Academy of Integrative Medicine, Dalian Medical University, Dalian, 116044, China.

${ }^{2}$ Laboratory of Pharmaceutical Resource Discovery, Dalian Institute of Chemical Physics, Chinese Academy of Sciences, Dalian, China. ${ }^{3}$ College of Pharmacy, Jinan University, Guangzhou, 510632, China.

${ }^{4}$ To whom correspondence should be addressed. (e-mail: maxc1978@163.com)
} 